\title{
Editorial: Current and Future Trends in Wireless Communications Protocols and Technologies
}

\author{
Muhammad Alam ${ }^{1} \cdot$ Mian Ahmad Jan ${ }^{2} \cdot$ Lei Shu ${ }^{3} \cdot{\text { Xiangjian } \mathrm{He}^{4} \cdot \text { Yuanfang Chen }}^{5}$
}

Published online: 8 March 2018

(C) Springer Science+Business Media, LLC, part of Springer Nature 2018

Information and Communications Technology (ICT) has significantly progressed in recent years by relying heavily on wireless communications. Currently, most of the devices and systems are connected via wireless technologies and numerous emerging solutions and applications are proposed based on wireless communications. Further, with the introduction of concepts such as Internet of Things (IoTs), nearly countless number of devices will be connected in a global network through wireless interfaces, using standard protocol solutions. The connectivity of these plethora of devices will be heavily relying on wireless communications and therefore, the challenges such as scalability, reliability, signal coexistence, data rate and energy consumption faced by the existing systems need to be thoroughly analyzed. In addition, the proposal for $5 \mathrm{G}$ technologies aims to provide very high data-rates, massive number of devices connectivity, very high reliability and low latencies. Therefore, efforts are underway to consider beyond state of-the-art protocols and mechanisms for wireless communication. Thus, the next generation of wireless communication is expected to meet the demands of various challenging use cases that go far beyond distribution of voice, video and data. Therefore, this special issue aims to present the solutions that address the challenges wireless communication systems are facing.

Therefore, European Alliance for Innovation (EAI) took a step towards the realization of Future Intelligent

Muhammad Alam

alam@av.it.pt

1 Instituto de Telecomunicações-Aveiro Campus, Universitário de Santiago, 3810-193, Aveiro, Portugal

2 Abdul Wali Khan University Mardan, Mardan, Pakistan

3 Guangdong University of Petrochemical Technology, Maoming, China

4 University of Technology Sydney, Ultimo, Australia

5 School of Cyberspace, Hangzhou Dianzi University, Hangzhou, China
Vehicular Technologies based on dependable and real-time communication and invite both academic and industrial research community by organizing the 2 nd edition of Future $5 \mathrm{~V}$ conference in Islamabad, Pakistan. Future $5 \mathrm{~V}$ is an annual international conference by EAI (European Alliance for Innovation) and co-sponsored by Springer. Future $5 \mathrm{~V}$ received more than 150 research articles in field of Vehicular networks/communications covering theory and practices in the after mentioned field of study. The call-forpapers of this SI was an outcome of the 2st EAI International Conference on Future Intelligent Vehicular Technologies and open submission. Following are the details of the accepted papers in this special issue.

The first paper is "The Model Design of Mobile Resource Scheduling in Large Scale Activities" by Cai-Qiu Zhou et al. In this paper, the authors have presented work on multiresource scheduling model based on Dijkstra and multiant colony optimization algorithms. The concept model of mobile resource scheduling is put forward, and the detailed introduction of each dimension is presented and multiresource scheduling model based on Dijkstra and multi-ant colony optimization algorithms are established respectively. Furthermore, the advantages and disadvantages of the two algorithms are compared through the numerical examples. It has been presented that Dijkstra algorithm is superior to multi-ant colony optimization algorithm in the cost control, but in the running time of the algorithm, multi-ant colony optimization algorithm is better than Dijkstra algorithm. The research has practical significance for the development of scientific and effective population service operation plan and service management plan for large scale exhibition activities.

The next accepted paper is "A Novel On-Line Association Algorithm for Supporting Load Balancing in MultipleAP Wireless LAN" by Liang Sun et al. It has been presented that wireless LAN has become the most widely deployed technology in mobile devices for providing Internet access. Operators and service providers remarkably increase the density of wireless access points in order to provide their 
subscribers with better connectivity and user experience. As a result, WLAN users usually find themselves covered by multiple access points and have to decide which one to associate with. The authors have proposed a novel online association algorithm to deal with any sequence of STAs during a long-term time such as one day. The performance of the proposed algorithm is evaluated through simulation and experiments. Simulation results show that our algorithm improves the overall WLAN throughput by up to $37 \%$, compared with the conventional RSSI-based approach. The presented algorithm also performs better than SSF (Strongest Signal First) and LAB (Largest Available Bandwidth) in the experiments.

The next paper is "GCC: Group-based CSI Feedback Compression for MU-MIMO Networks" by Jian Fang et al. The Multi-user Multiple Input Multiple Output networks (MU-MIMO) adopts beamforming to enable Access Point (AP) to transmit packets concurrently to multiple users, which brings formidable overhead. The overhead of collecting Channel State Information (CSI) feedback matrix may even overwhelm real-data transmission when the scale of network is large, which incurs unsatisfactory performance and huge waste of resources. Therefore, in this paper, the authors have addressed this problem using GCC which is a Group-based CSI feedback Compression scheme for MU-MIMO networks. It enables users to feedback their CSI in terms of group determined by their location. The GCC limit the quantity of CSI feedback in each transmission round regardless of the size of network by allowing the location-related users to share a CSI matrix. In addition, the authors have used a novel metric to do the tradeoff between throughput and capacity loss of the system. GCC has been tested in different scenarios and compared it with existing works. The evaluation result showed that GCC achieved much higher throughput and is robust to various situations.

Since the The growth and adoption of the Internet of Things (IoT) is increasing day by day and it has become a very hot research topic. We have considered a paper for publication that has presented a Framework for trust management in IoT. The paper is tittled "Clustering-Driven Intelligent Trust Management Methodology for The Internet of Things" by Mohammad Dahman Alshehri et al. One possible approach to achieve IoT security is to enable a trustworthy IoT environment in IoT wherein the interactions are based on the trust value of the communicating nodes. The authors have proposed a methodology for scalable trust management solution in the IoT. The methodology addresses practical and pressing issues related to IoT trust management such as trust-based IoT clustering, intelligent methods for countering bad-mouthing attacks on trust systems, issues of memory-efficient trust computation and trust-based migration of IoT nodes from one cluster to another. Experimental results demonstrate the effectiveness of the proposed approaches.

A comprehensive study on designing an energy-aware architectures for Wireless Sensor Networks is presented in the next accepted paper. The paper is "Designing an EnergyAware Mechanism for Lifetime Improvement of Wireless Sensor Networks: A Comprehensive Study". The authors have also analysed and proposed a scheme, ExtendedMultilayer Cluster Designing Algorithm (E-MCDA) in a large network. Among its components, algorithms for time slot allocation, minimising the $\mathrm{CH}$ competition candidates, and cluster member selection to $\mathrm{CH}$ play underpinning roles to achieve the target. The authors have done simulations in NS2 to evaluate the performance of E-MCDA in energy consumption at various aspects of energy, packets transmission, the number of designed clusters, the number of nodes per cluster and unclustered nodes. It is found that the proposed mechanism optimistically outperforms the competition with MCDA and EADUC.

The next paper is "Rule based (Forward Chaining/Data Driven) Expert System for Node Level Congestion Handling in Opportunistic Network" by Ahthasham Sajid et al. The paper is about Opportunistic networks which are part of the most popular categories of Mobile Ad hoc networks. One of the challenge is the selection of best custodian node that can store messages at its buffer until a destination node is found. The important features of the Delay Tolerant Network (DTN) are a selection of the best forwarding nodes and co-ordination among the nodes to deliver the packets to their destination in an efficient manner with less loss and maximum delivery rate. Therefore, in this paper, the authors have presented a rule based efficient expert system to address and handle storage level congestion issues. The proposed technique has been tested and validated via Opportunistic network environment and compared with MaxProp protocol.

The next paper is "A Comprehensive Analysis of Congestion Control Protocols in Wireless Sensor Networks" by Mian Ahmad Jan et al. In wireless Sensor Networks (WSNs) congestion occurs when the incoming traffic load exceeds the available capacity of the network. The authors have also presented the various factors that lead to congestion in WSNs such as buffer overflow, varying rates of transmission, many-to-one communication paradigm, channel contention and the dynamic nature of a transmission channel. The energy-efficient congestion control protocols need to be designed to detect, notify and control congestion effectively. The authors have present a review of the latest state-of-the-art congestion control protocols. Depending on their inherent nature of control mechanism, these protocols are classified into three categories, i.e., traffic-based, resource-based and hybrid. Traffic-based protocols are further subdivided, based on their hop-by-hop or 
end-to-end delivery modes. Resource-based control protocols are further analyzed, based on their route establishment approach and efficient bandwidth utilization techniques. In addition, they have discussed the internal operational mechanism of these protocols for congestion alleviation. The authors have concluded that the behaviour of each class of protocols varies with the type of application and a single metric alone cannot precisely detect congestion of the network.

In the last decade, there has been a considerable development in the field of wireless vehicular communications so as to satisfy the requirements of Cooperative Intelligent Transportation Systems (CITS). It is also worth mentioning that currently CITS and intelligent transportation systems are very hot research areas and especially the security of these systems. We have accepted the paper "Implementation and Analysis of IEEE and ETSI Security Standards for Vehicular Communications" by Bruno Fernandes et al. The paper presents the implementation and analysis of the two most used standards for vehicular communications. However, due to the expected popularity of ITS, VANETs could be prone to attacks by malicious sources. To prevent this, security standards, such as IEEE 1609.2 and ETSI ITS' standards, were developed. In this work, the design and implementation of an API capable of conducting the required cryptographic algorithms and protocols for the transmission of secure messages according to the IEEE 1609.2 and ETSI ITS' security standards is presented. The implemented security protocols are then integrated into a system emulating a public key infrastructure to evaluate the performance impact on safety-related communications, in particular, the delay associated with the communication' coding/decoding process.

The next paper is "Performance of Cognitive Radio Sense-and-Wait assisted Hybrid Automatic Repeat reQuest" by Fazlullah Khan et al. In this paper the authors have presented a work on the the cognitive radio (CR) concept which emerges as a promising solution for reducing the spectrum scarcity issue. The CR network is a low cost solution for efficient utilization of the spectrum by allowing secondary users (SUs) to exploit the unoccupied licensed spectrum. The authors have presented the model the PU's utilization activity by a two-state Discrete-TimeMarkov Chain (DTMC) (i.e., Free and busy states), for identifying the temporarily unoccupied spectrum bands,. Furthermore, they have proposed a Cognitive Radio Senseand-Wait assisted HARQ scheme, which enables the Cluster Head $(\mathrm{CH})$ to perform sensing operation for the sake of determining the PU's activity. Once the channel is found in free state, the $\mathrm{CH}$ advertise control signals to the member nodes for data transmission relying on Stopand-Wait Hybrid- Automatic Repeat-Request (SW-HARQ). The proposed CRSW assisted HARQ scheme is analytical modeled, based on which the closed-form expressions are derived both for average block delay and throughput. Finally, the correctness of the closed-form expressions are confirmed by the simulation results.

The next accepted paper is "Opportunistic Energy Cooperation Mechanism for Large Internet of Things" by Jinyu $\mathrm{Hu}$ et al. In this paper, the authors have focus on energy efficiency maximization and network throughput optimization problems for energy cooperation in Energy Harvesting Cooperative Wireless Sensor Networks (EHC-WSNs). In order to maximize the efficiency of energy charging phase, a Region-based Proactive Energy Cooperation (RPEC) strategy is developed, which is used to charge the life-critical cooperators or receivers in time. Furthermore, by introducing a novel metric that converts optimal forwarder selection from the multidimensional problem to one-dimensional problem, an Energy-Neutral based Opportunistic Cooperative Routing (ENOCR) algorithm is proposed to optimize the relay nodes selection and improve the network throughput. The simulations results showed that the proposed mechanism can significantly improve energy efficiency and network lifetime.

The last paper is "User-centric Clustering and Beamforming for Energy Efficiency Optimization in CloudRAN". In this paper, the authors have considered the problem of how to assign each user to several preferred remote radio heads (RRHs) and design the corresponding beamforming coefficients in a user-centric and energy efficient manner. They have formulated the problem as a joint clustering and beamforming optimization problem, with the objective to maximize the energy efficiency (EE) while satisfying the users' quality of service (QoS) requirement and respecting the RRHs' transmit power limits. They have first transform it into an equivalent parametric subtractive problem using the approach in fractional programming, and then it is cast into a tractable convex optimization problem by introducing a lower bound of the objective function. Finally, the structure of the optimal solution is derived and a two-tier iterative scheme is developed to find the clustering pattern and beamforming coefficients that maximize EE. Specially, they have derived a RRH-user association threshold, based on which the RRH clustering pattern and the corresponding beamforming coefficients can be simultaneously determined. 


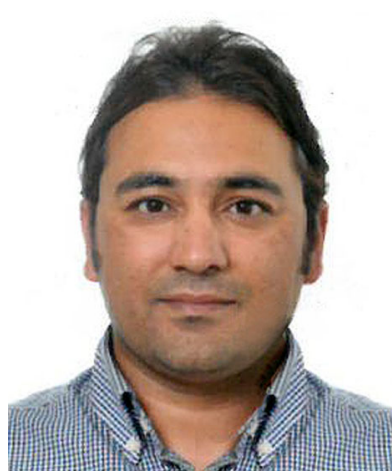

Muhammad Alam holds a $\mathrm{PhD}$ degree in computer science from University of Aveiro, Portugal (2013 - 14). In 2009 , he joined the Instituto de Telecomunicações - Aveiro (Portugal) as researcher and completed his Ph.D. from University of Aveiro. He has participated in several European Union FP7 projects such as Hurricane, C2POWER, ICSI, PEACE and Portuguese government funded projects such SmartVision. Currently, he is working as senior researcher at Instituto de Telecomunicações and participating in European Union and Portuguese government funded projects. His research interests include Real-time wireless communication, 5G, Vehicular networks, Context-aware systems and Radio resource management in next generation wireless networks. He is the author of several journal and conference publications as well as book chapters. He is also the TPC member and reviewer for a number of reputed conferences, journals, and magazines. He is IEEE senior member. He served as general cochair of future $5 \mathrm{~V}$ conference and also served as session chairs in a number of conferences.

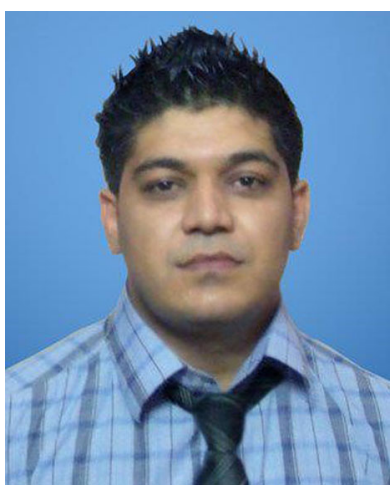

Mian Ahmad Jan is an Assistant Professor at the Department of Computer Science, Abdul Wali Khan University Mardan, Pakistan. He holds a $\mathrm{Ph} . \mathrm{D}$. in Computer Systems from University of Technology Sydney, Australia. His research interests include clusterbased hierarchical routing protocols, congestion detection and mitigation and intrusion and malicious attack detections in wireless sensor networks, Internet and web of things. He has published his research in various prestigious IEEE Transactions, Springer and Elsevier Journals. Besides, he has published his work in numerous International Conferences as well. He has served on editorial board and guest editor for various prestigious journals. $\mathrm{He}$ is an active reviewer of numerous journals.

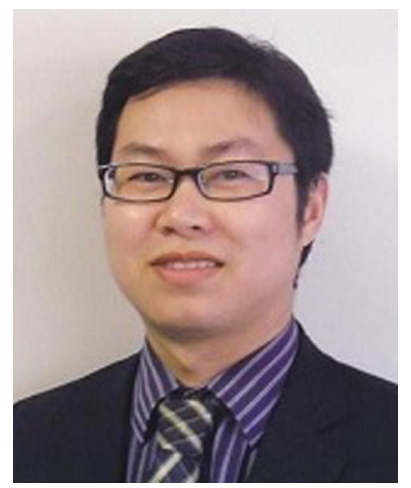

Lei Shu received B.Sc. degree in Computer Science from South Central University for Nationalities, China, in 2002 and M.Sc. degree in Computer Engineering from Kyung Hee University, Korea, in 2005 and the Ph.D. degree in Digital Enterprise Research Institute, from National University of Ireland, Galway, Ireland, in 2010. He is member of IEEE, IEEE IES, IEEE ComSoc, EAI and ACM. Since October 2012, he joined Guangdong University of Petrochemical Technology, China as a full professor. His research interests include: Wireless Sensor Networks, Multimedia Communication, Middleware, Fault Diagnosis, and Security and have published over 230 papers in related conferences, journals, and books in the area of sensor networks. $\mathrm{He}$ is active in various research fields in computer science domain and associated with a number of conferences and journals.

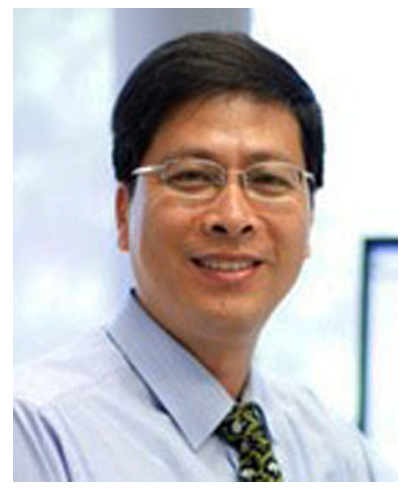

Xiangjian He is a Professor of Computer Science, School of Computing and Communications. He is also Director of Computer Vision and Pattern Recognition group, and a Deputy Director of Research Centre for Innovation in IT Services and Applications (iNEXT) at the University of Technology, Sydney (UTS). $\mathrm{He}$ is an IEEE Senior Member. $\mathrm{He}$ has been awarded 'Internationally Registered Technology Specialist' by International Technology Institute (ITI). He has received many competitive national or regional grants including four grants awarded by Australian Research Council (ARC), two grants awarded by National Natural Science Foundation of China (NSFC), and one grant awarded by Hong Kong Research Grants Council (RGC). His research interests are image processing, pattern recognition, computer vision and Network security. He is in the editorial boards of seven international journals. He has received various research grants including four national Research Grants awarded by Australian Research Council (ARC). 


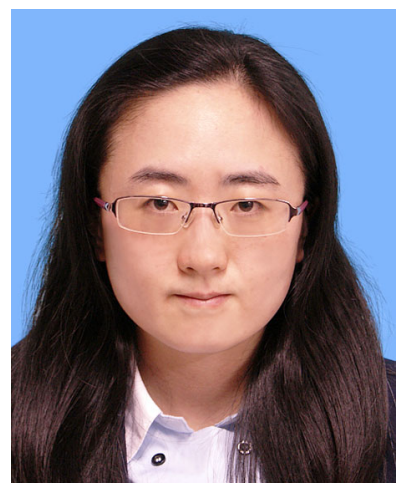

Yuanfang Chen is currently a Distinguished Professor with the Hangzhou Dianzi University, China. She received the M.S. and the first Ph.D. degrees from the Dalian University of Technology, China, and the second Ph.D. degree from the University Pierre and Marie CURIE, France. From 2009 to 2010, she was an Assistant Researcher with the Illinois Institute of Technology, USA, with Prof. Xiangyang Li. Her research interests include localization, deep/machine learning, algorithm design, and wireless sensor networks. She was a Travel Grant recipient of MASS 2009, IWCMC 2009, and SIGCOMM 2013. She has served as a volunteer of MobiCom 2010 and MobiHoc 2010, and IEA-AIE 2012. She has been invited as the Session Chair of conferences, including MobiQuitous 2013, ICA3PP 2015, and CODIT 2018, and the TPC member of Globecom 2014 and MobiApps 2014. She has served as the Fixed Reviewer of top journals and conferences, including IEEE TRANSACTIONS ON INDUSTRIAL INFORMATICS, ACM Computing Surveys, IEEE TRANSACTIONS ON FUZZY SYSTEMS, and AD HOC and; SENSOR WIRELESS NETWORKS. She is currently an Associate Editor of the EAI Endorsed Transactions on Industrial Networks and Intelligent Systems. 